\title{
El demonio de La Paz
}

Hay otros mundos, pero

están en éste.

Eluard

En este artículo ensayo una interpretación de "El centauro criollo", un relato propio del departamento de La Paz, El Salvador, recogido por el escritor Rafael Rodríguez Díaz en su compilación crítica Los duendes de la barranca honda' . Se trata de una historia que se transmite de forma oral entre los lugareños, y que ha sido recogida —en los primeros años de la postguerra salvadoreña-, transcrila y ordenada por el compilador. Me limitaré a anotar algunas observaciones que permitan un acercamiento a los elementos naturales y culturales propios de la vida cotidiana de los pobladores de La Paz, con lo cual espero penetrar en la identidad de este grupo humano que ha proyectado en un relato mítico su conciencia colectiva, la cual, probablemente, tendría mucho en común con la de otros grupos salvadoreños ${ }^{2}$.

En un primer momento tenemos la "historia", la trama que hay que analizar, para lo cual transcribo integramente el texto de Rafael Rodríguez ${ }^{3}$. Luego, procederé con la aplicación de dos métodos de análisis de los relatos míticos: por una parte aplico la metodología de Tzvetan Todorov para el análisis de las secuencias presentes en la trama^; por otro lado, el método de Claude LéviStrauss me permite entrar en el problema de las correspondencias entre los elementos del cuento y las caraclerísticas de la vida del grupo étnico que lo origi$\mathrm{na}^{5}$. Al final, muestro dos interpretaciones del mito: la realizada por el compilador, de la cual discrepo en buena medida, y la mía.

\section{El hombre-caballo}

Una alumna de la UCA, Susana Reyes, fue la primera en hablame del extraño animal:

Fijese que mi mamá va lodos los días a San Juan Tepezontes; ahí trabaia en 
la Unidad de Salud... Pues, en San Juan Tepezontes han visto a una especie de centauro: un animal mitad caballo y mitad gente.

Hace poco un señor iba en su bestia llevando unas reses para su terreno, cuando en eso oyó que detrás de él venía un caballo en una gran carrera. Sin volliar a ver se apartó para dejar que pasara el animal o el jinete. Pero, al momentito, ya no oyó nada.

Siguió su camino el señor y al poco rato, otra vez la gran carrera que se oía detrás de él... Ahora sí voltió la vista y alcanzó a ver que un hombre con cuerpo de caballo se tiró a esconderse en unos charrales, al lado del camino... El señor llegó con unas grandes calenturas a su casa y sin poder hablar.

Intrigado por la noticia, continué indagando sobre ese espécimen de nuestra zoología fantástica. Inesperadamente, fue en la Costa del Sol, en el terreno de los Melhado, donde escuché más dalos curiosos. Walter, un cipote como de trece años que ayuda en los quehaceres a la familia Melhado, fue mi informante:

Ya lo han visto por aquí, por San Marcelino. A un señor y a una señora que se les apareció se los llevaron al hospital porque se habían quedado sin poderse mover y sin poder hablar...

Una mañana, Oscar y Araminta me invitaron a viajar desde su terreno, en la Costa del Sol, hacia la zona de los Tepezontes: a ver qué averiguábamos sobre el extraño aparecido... Llegamos hasta San Pedro Masahuat, pero no se nos ocurrí́ preguntar nada ahí, porque en ese enlonces no sabíamos que San Pedro también era escenario de las apariciones del centauro. Así que la expedición hacia los Tepezontes no se culminó, ni tampoco pudimos averiguar más datos nuevos sobre el aparecido.

Los comentarios sueltos de una señora que vendía queso y crema en la desembocadura del río Jiboa, parecían dar por concluido el capítulo sobre el hombre-caballo; pero una cinta grabada por otra alumna mía, Silvia Araniva, vendría a completar maravillosamente la información que me faltaba.

Silvia grabó la conversación con un señor que vive en las proximidades de San Pedro Masahual, sobre la calle que, yendo hacia el sur, pasa por Dulce Nombre y va a desembocar a la carretera del Litoral. Esa misma calle de tierra, hacia el norte, pasa por San Pedro y desemboca en la carrelera vieja, en las proximidades de San Miguel y San Juan Tepezontes.

En cuanto al relato, he querido hacer sólo unos pequeños retoques (orden de los hechos, supresión de partes confusas o repetitivas), conservando en la medida de lo posible el estilo del informante. Los giros y expresiones propios del estilo de dicho señor hacen del relato algo muy ameno, al par que riquísimo en información antropológica.

Aquí en San Pedro, en el desvío que va para Dulce Nombre..., ha aparecido un animal feyo, dice la gente que lo ha vislo... De la cintura para'rriba es 
hombre, y de para'bajo..., es forma de caballo. Aparece desde las once de la noche para allá. Ahí por el pantión degolló a unos chuchos...; porque degolla chuchos, patos, gallinas. No mira que a un señor le dogolló los gansos que tenía..., y siguió para'rriba.

Dicen que va para los Tepezontes; para'allá dicen que va caminando... Pero aquí en San Pedro toda la gente, todo el mundo tiene miedo. Por eso es que en la escuela han dado escapularios: para que se cuiden, porque los bichilos tienen miedo de que los vayan a asustar o a llevar... Y es que también a los niños recién nacidos que no están bautizados se los lleva; los degolla o no sé si les hace algún mal. A saber cómo serán esas cosas malas... Ah, pues, y de ahí a un señor le estaba matando al chucho..., y encendió la luz. Cuando encendió la luz quedó paralítico... Y otro señor que vive por ahí abajito había echado agua bendita alrededor de la casa, porque tiene un chuchito y no quiere que se lo lleve.

Aquí está feyo. ¡Dios guarde si alguien se atreve a salir de noche! ¡Huy, no puede salir...! Dicen que era un chamaco, un bato que estaba practicando brujerías y, entonces, una noche dejó el alma en un vaso de agua y salió... En la mañana, la mamá, sin saber, botó el agua..., y por eso el chamaco se quedó en forma de animal... Como hay quienes les gusta andar chingando; por eso, este baboso se quedó en forma de animal..., y por eso anda penando. Dicen que tiene que matar más de doscientos chuchos -algo así; no me acuerdo- para volver a su vida real..., y cuatro corazones de niños se tiene que comer..., de niños tiemitos, sin bautizar...

Dicen que viene de Usulután; por todo eso pasa..., por el Rosario. Cuando caminó para'llá bajo, más abajo de por aquí del desvío de Dulce Nombre, para'bajo, para Barahona..., por ahí, en una milpa, un señor lo vio revolcándose en todas esas matas de maíz. El señor con el corvo y su hijo..., siguieron al hombre. Iba echando carcajadas bien feyas y desapareció. Porque corre bien duro, duro, duro... El señor iba corriendo con su caballo, iba siguiéndolo. Y el hombre coría más duro..., y se metió en unas cuevas, por unos matorrales...; por eso la gente ya no lo ve.

Hace poquito un padre de Barahona dicen que lo agarró..., con cadenas lo tenían. Y dicen que montón de gente iba a ver eso, porque pensaban que lo iban a matar...; pero no lo pudieron quemar porque se soltó, y todavía sigue por ahí.

Aquí a cada rato pasan males... Si por todo eso hay monión de cosas que han sucedido. Si aquí usted le pregunta a un baboso: '-Mire, ¿es cierto esto?', le va a decir que sí, porque aquí es verdad lodo eso... Si aquí ha pasado; nosotros lo hemos vislo. Si en la noche, una aullazón de chuchos... ¡Uuuhhh...! ¡Cómo gritan cuando les degollan el cuello! Y todo feyo amanece, todo callado. 
Aquí por el cantón, un poquito más abajito, una noche aparecieron las calles ensangrentadas, llenas de sangre..., un montón de sangre regada..., y una yedazón que había, un tufo... ¡Ujuummm!, iqué bárbaro!, un tufo bien feyo. Asco daba; ganas de arrojar. Cabezas de chucho por ahi, patas, pescuezos... Las gallinas, las patas por un lado. Dicen que a las gallinas se las come vivas..., las cabezas se come primero. Por eso es que uno no sale aquí en la noche.

Dicen que hoy luego lo han visto por los Tepezontes..., que va subiendo esos cerros... Hace poco vino olra vez por aquí. Vino a hacer olro desvergue y se fue... La cosa es que a saber si va a volver.

La gente ya sabe, está sabida de que si llegan a matar a un niño y a quitarle el corazón..., ya saben a la señora que van a'garrar porque es la mamá... Ella anda buscando los corazones; anda matando a los niños para quitarles el corazón y así llevárselos.

Lo que's por este desvío de Dulce Nombre para'rriba ha pasado. Dicen que ha bajado ahí para Tapalhuaca..., un poquito más allá; quizás medio kilómeIro de donde dicen Comalapa, donde hay una gasolinera. Por ahí sube...; dicen que por ahí lo han visto salir... También ha hecho matazón. Dicen que vivas se come a la gallinas

Y dicen - a saber, a mí no me consta - que ha llegado hasta Olocuilta; que en Cuyultitán lo vio una señora y quedó también paralítica, sin poderse mover del susto, bien espantada. Y ahí por el cementerio de Olocuilta, a una muchacha como de quince años dicen que le salió; pero ella no se asustó, y se fue corriendo el animal... Como dicen que ahí hay un montón de monte en una quebrada, ahí se metió...

Cuando lo fueron a buscar con corvos, macheles, cumas, lodas esas ondas, lo hallaron que ya se había convertido en persona, y no le quisieron pegar su cutazo porque pensaron que quizás era genle. Pero después dijeron que era el baboso que anda...

Creo que quizás en el día pierde su poder; pero en la noche, en la oscuridad, dicen que tiene unos ojos que brillan... El pecho todo peludo; la cabeza, llena de pelos. Bien peludo, peludo, peludo..., y la cola. Todo feyo el cuerpo... El tipo es negro..., o a saber de qué color es; gris ha de ser el baboso... Y ahí anda asustando a la gente. Por eso, mi mamá me ha dicho que siempre rece en la noche. Y si voy a salir a todo eso, a las diez de la noche... Pero hoy ni nos movemos de aquí porque tenemos miedo que vaya a llegar a tocar la puerta.

Como en octubre o septiembre fue todo esto; pero parece que ha vuelto el baboso. Por eso, mi mami me ha dicho que recemos antes de dormir y que no nos quedemos muy noche y no andemos haciendo cosa malas...; porque 
dice ella que ha salido en la Biblia que se verán cosas muy malas. Por eso, hay que portarse bien y hay que ser buenos...; así me ha dicho mi mamá.

Interrumpo el relato para consignar lo que el amigo Pablo Morales me ha informado al respecto. Pablo visita con frecuencia un cantón que queda más o menos por la misma zona donde vive el informante. También a Pablo le han contado sobre el extraño animal..., y le han dicho que el centauro ha proferido algo así como una sentencia bíblica..., relativa a los niños. Y la grabación continúa:

Ahí en Tapalhuaca, dicen que a una muchacha, una noche, le fue a tocar la puerta..., como iba a tener un niño..., estaba embarazada. Por eso, ella se fue para donde una lía..., porque no vaya a ser que le hiciera algún mal.

Antes de subir a Olocuilta, dicen que pasó por el aeropuerto. Cuando iba pasando, los guardias de seguridad empezaron a disparar, y cuando tiraron el primer tiro..., se fue la luz en el aeropuerto... La gente dice que es cosa del diablo que anda ahí..., que el diablo hizo que apagaran las luces para que no le hicieran nada... Sí, porque le tiraron y ni uno le pegaron... Después fue que subió a Olocuilta.

Otro dato más me obliga a una nueva interrupción. Susana Reyes me contó que cuando el centauro se iba acercando al aeropuerto, efectivamente, se fueron las luces, pero un señor logró iluminarlo con los faroles de su automóvil y alcanzó a ver cuando el centauro se tiraba por unos montarrascales, al lado de la carretera. Y el informante sigue:

Al señor que echó agua bendita, también a él le llegó a tocar la pucria. Por eso, nosotros tenemos agua afuera de la puerla; porque dice Papa Tin que el agua, cuando a uno le tiran un mal en la noche, caye'n el agua..., y como es agua bendita, no nos hace nada. Eso nos ayuda...; asi dice Papa Tin. Por eso es que nosotros agua ponemos en la noche...: para que si nos tiran un mal o viene el baboso, no nos hagan nada.

Yo por aqui digo que no le tengo miedo, pero a la hora de las horas yo creo que me le agüevo a ese baboso... ¡Ay, Dios...!, con que ni a mis chuchos le echaba, porque ipobrecitos mis chuchos! ¡Ah, y nos mató a un chucho a nosotros también...! ¡Un chucho tan bonito...! Llegó en la noche; todos estábamos en silencio; agarró al chucho... ¡Auuuhhhh!, se oyó un grito bien feyo y lo degolló. Cuando amaneció, la cabeza la tenía trabada en un palo...; las patas por allá, $y$ todo eso... ¡Ujuuummm!

Mucha gente dice que lo ha visto...; pero los únicos que lo pueden ver bien son los chuchos y los brujos... Hay unos brujos aquí que dicen que to han visto..., porque uno no lo puede ver... Tampoco a los brujos se les puede ver. Para decirle que yo no conozco a ningún brujo, porque es que los brujos se 
ven gente común, pero saben brujerías... Dicen que para andar en la noche y que no le salga tanto ese mal, hay que fumarse un puro... Los que saben de esas cosas, eso hacen y no les pasa nada; pero uno así como yo, ¿cómo voy a estar tranquilo andando tipo ocho de la noche, yéndome para mi casa...? Yo vivo como a tres kilómetros del desvío para'rriba; ahí por la calle. Ahí para el Dulce Nombre para'riba...; ahí vivo yo, ahí estoy...

Bueno..., si les quiere decir a las demás gentes que tengan cuidado, porque no se sabe si va a volver... Tal vez a estos lugares porque están aislados... Pero nadie se tiene que confiar de esa cosa; porque supóngase que vaya a las ciudades. Allá es cierto que pasan las puertas cerradas..., pero también puede llegar...; no sólo aquí en el cantón...

Aquí han venido periodistas...; aquí han venido a preguntar gentes de otras partes, que si es cierto..., y nosotros les decimos que sí, porque sí es cierto... Ya es tarde y tengo que ir a traer lo que le dije... Adiós, pué..." (p. 10-11) .

\section{"El centauro criollo" como relato de ley}

Tralando de rescatar aquellos elementos suficientes para la consirucción de un modelo de interpretación tenemos:

- un joven practica brujería

- esto implica quebrantar las leyes de Dios

- luego desobedece una regla implícita de los brujos: la precaución

- es castigado: se transforma en monstruo

- emprende una serie de acciones que buscan revertir el castigo: sacrificios humanos y animales

- el asunto queda abierto, ya que las apariciones continúan.

En el relato encontramos el esquema básico del relato de ley que propone Todorov:

$$
\text { quebranto de la ley } \rightarrow \text { acción que busca evitar el castigo }
$$

que podernos reducir aún más al siguiente esquema formal:

$$
X b(\Rightarrow Y c X) \rightarrow(X-c X) \Rightarrow X a \Rightarrow Y-c X
$$

en donde:

$X, Y$ son los agentes, los "personajes" principales en el relato

$b$ es una transgresión de la ley (implícita o explícita)

$c$ es el castigo por llegar

$a$ es la acción necesaria para evitar el castigo

() indican la pura posibilidad. 
Haciendo las sustituciones pertinentes tenemos:

brujeria $\rightarrow$ descuido $\Rightarrow$ metamorfosis $\rightarrow$ revertir $\Rightarrow$ comer corazones/sacrificios $\Rightarrow$ ?

Como podemos ver, se nos presentan algunas variaciones, ya que de la infracción de la ley que implica la brujería pasamos a la infracción por el descuido; por otro lado, el castigo se hace efectivo (nótese que no hemos colocado los paréntesis) y no existe la restitución del orden quebrantado: la metamorfosis yo ha sido efectuada (el hombre-caballo monstruoso) y los sacrificios realizados parecen no sener fin.

Ahora bien, no obstante las diferencias, creo posible explicar el relato como un conjunto de sucesos atemorizantes que giran en tomo a los actos de aquél que busca revertir las consecuencias de su transgresión de la ley. La relación quebranto de la ley $\rightarrow$ acción que busca evitar el castigo se verifica en nuestro caso bajo la forma más precisa de pacto con el Diablo $\rightarrow$ sacrificios humanos. Más adelante retomaremos estas ideas.

\section{Los niveles del análisis antropológico en la historia}

Lévi-Strauss propone cuatro niveles que permiten ordenar e interrelacionar los diversos elementos constituyentes del contexto vital del grupo humano que ha creado el relato o mito. Estos son: geográfico, económico, social y cosmológico. Como puede verse, se toman en cuenta tanto los aspectos naturales como los culturales. Es más, el método de Lévi-Strauss pretende relacionar estos elementos entre sí y encontrar los esquemas o contenido latente que permita conectarlos y, a la vez, sintetizarlos en un elemento que permita ver con claridad el significado que el grupo ha producido y que lo identifica.

Yo he modificado, aunque no sustancialmente, los niveles del análisis, lo cual me permite obtener, a partir de las apariciones del hombre-caballo, los siguientes esquemas de oposiciones:

Nivel espacio-lemporal:

liempo/hora:

$$
\text { Día }
$$

Noche

local/sitio:

Casa

Camino

región/zona:

Tierras bajas

Tierras altas

dirección/geografía:

Sur-occidente

Norte-oriente 
Las apariciones del hombre-caballo se suceden en la noche, preferiblemente en los caminos. Está claro también que se trata de una bestia que baja de los montes, de las tierras altas (Los Tepezontes), y es ahí a donde se dirige una vez cometidas sus fechorías. En una ocasión se menciona además que es originario de Usulután; si a esto añadimos que sus desplazamientos dentro de la zona de las apariciones son desde el noreste al suroeste (de Los Tepezontes hacia San Pedro Masahuat, de este pueblo hacia Olocuilta, de la zona mencionada hacia el litoral, etc.), podemos suponer que su lugar de procedencia es el norte-oriente.

Nivel laboral:

\section{Campesino}

Cazador-"Sacrificador"

El hombre-caballo se dedica a la caza de animales y a la matanza sacrificial de seres humanos inocentes - niños-, lo cual es un elemento opuesto a la tranquila y cristiana? existencia de los campesinos de la zona.

Nivel sociológico:

$$
\text { Lugareño }
$$

Extraño

Hay diversas alusiones a que "el animal" es un extraño, alguien que no es de esos lugares.

$\left[\begin{array}{r}\text { Nivel moral-ontoló } \\ \text { Bien-Vida } \\ \text { Nivel cosmológico: }\end{array}\right.$

"Gente común"

Mal-Muerte violenta

El comportamiento bueno se expresa en el estilo de vida tradicional y alejado de las "cosas extrañas": el mal moral no está desconectado del Mal ontológico. Pero esto se encuentra íntimamente relacionado con el orden del universo que se supone en el relato: no cualquiera puede "ver" bien a la bestia, sino el que pertenece a su "esfera natural"; sólo los brujos pertenecen al "mundo" del hombre-caballo: la magia negra.

Basta con echar una mirada a la narración para encontrar los diferentes elementos que aquí menciono. También, dado que el relato se encuentra en un nivel muy primario de elaboración y, además, ya que los "hechos" que se narran son recientes, podemos presumir una correlación entre los datos sobre la vida de los "personajes" involucrados en el cuento y la forma de vida del grupo humano concrelo que lo ha producido. 
Pero nuestro trabajo no termina aquí, ya que es preciso reducir nuestras oposiciones al significado global del cuento. Si tomamos cada uno de los esquemas anteriores tenemos un esquema cuádruple que expresa una oposición integradora:

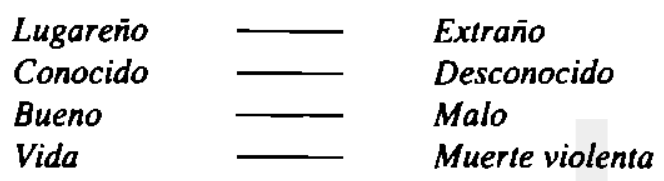

Es así que podemos colocar en un mismo grupo los componentes tales como dia, casa, tierras bajas, suroeste, campesino, lugareño, bien-vida, "gente común" y, por otra parte, a sus opuestos: noche, camino, tierras altas, noreste, cazador-"sacrificador", extraño, mal-muerte violenta, brujos, sin anular sus particulares significados, a la vez que obtenemos una explicación coherente y sintética de las relaciones de oposición entre ellos.

Entonces, y como último paso en nuestro proceso hermenéulico, obtenemos una oposición que viene a dar sentido a todo el relato, tomando el primer componente positivo en nuestro esquema y el último de los negativos:

\section{Lugareño $\quad$ Muerte violenta}

Según lo anterior, la muerte violenta, que no tiene otro agente que El Mal, no pertenece a la cotidianidad del lugar, sino que es algo malo que viene de lejos. En el último apartado veremos cómo este significado se amplía al tomar en cuenta lo obtenido de la aplicación del método de análisis de Todorov, así como el contexto nacional en la época de las "apariciones".

\section{El "centauro criollo": ¿hibrido?}

Veamos ahora la interpretación que hace Rafael Rodríguez Díaz. Éste reloma el contexto histórico de los pobladores de la zona en cuestión, así como las opiniones más conocidas sobre los "centauros":

"Los nonualcos convivieron en Tula con los tollecas adoradores de Quetzalcóatl; pero, llegado un momento, los nonualcos quisieron imponer los sacrificios humanos como práctica habitual. A resultas de la querella fue expulsado Ce Acatl Topiltzin Quetzalcóatl, sacerdote del culto a Quetzalcóatl. Corrían los últimos años del siglo X. Los nonualcos permanecieron en Tula hasta el siglo XII; luego pasaron a otros lugares de México. Descendientes suyos fundaron el reino de Cuscatlán y se establecieron en lo que ahora es el departamento de La Paz.

Adentrándome en esa historia de los grupos que poblaron la zona mencionada en el relato, iba teniendo yo algunas pistas más reveladoras: esos grupos 
fueron tremendamente belicosos y aguerridos. Su práctica era el sacrificio humano, extrayendo el corazón de las víctimas (...) El centauro que se ha aparecido en la parte occidental del departamento de La Paz exige sacrificios de niños (debe extraerles el corazón) y eso lo asemeja a las prácticas de los antiguos pobladores de la región... (p. 11).

Para algunos intérpretes los centauros no son más que la corporización mítica de la tempestad. Cuando ésta arremete, pasa arasándolo todo como si fueran caballos (o centauros) al galope; y el hecho de que el huracán arranque los árboles de cuajo fue incorporado a la imagen de los centauros levantando en vilo enormes árboles para blandirlos o lanzarlos como armas contra sus enemigos. En suma, los centauros - asociados o no con la tempestad- eran vistos míticamente como guerreros bárbaros y despiadados, capaces de sembrar la destrucción por donde iban pasando (p. 12).

Según nuestro compilador y crítico, el relato se podría caracterizar como un mito de hibridación, ya que ésta es la idea que subyace, inlegrando la historia y los significados adjudicados por el grupo humano que la ha creado:

El centauro es un ser hibrido de persona y animal, ya lo sabemos; pero recordemos, además, que con la venida de los españoles se dio un proceso parecido a la formación (habida entre los griegos) del mito del centauro. Para los indígenas, los españoles que venían montados en sendos caballos formaban una sola entidad y una sola divinidad. Caballero y cabalgadura se impusieron, en las primeras de cambio para los indígenas, como un dios terrible y batallador. $Y$, aunque después los indígenas ya pudieron hacer perfectamente la diferencia entre jinete y caballo, la figura del centauro bien pudo haber quedado como imagen arquetípica en los sustratos de la cultura y de la conciencia indígenas. Es decir, ese ser monstruoso pasó a representar, de ahí en adelante, el temor ante una violenta e irrefrenable agresión desde el exterior.

El 'centauro criollo' haría referencia, consecuentemente, al componente europeo occidental que está inscrito en los estratos más profundos de nuestra in-conciencia colectiva. Pero como también dice el relato que el 'centauro criollo' degolla animales y está dispuesto a extraer los corazoncilos de cuatro niños tiernos, entonces representa la tendencia sacrificial propia de las sociedades indígenas nonualcas precolombinas.

El 'centauro criollo' resulta, así, un ser hibrido bajo varios respectos: lo es, primero, por su composición de humano y animal; pero lo es, en segundo lugar, por sus características mestizas; es decir: es un monstruo típicamente europeo, pero con un comportamiento lípicamente indígena (p. 12).

Pero el fenómeno va más allá de la mera remembranza, ya que la guerra civil salvadoreña acaba de terminar, por lo cual es de suponer que lo más negativo de la herencia indígena y española retorna para explicar el asedio de la violencia 
que se padece en estos difíciles tiempos de postguerra:

"Los Acuerdos de Paz, firmados en enero de 1992, cerraron las puertas a la guerra y las abrieron para la posibilidad de construir una nueva sociedad en paz y libertad. Sin embargo, no todo está marchando sobre ruedas: la anomia social, la pobreza extrema, la delincuencia descarada. Todo eso habla de la posibilidad del recrudecimiento de la violencia y de la vuelta a liempos de zozobra e incertidumbre.

$Y$ es en este sentido que impone su profundo simbolismo el 'centauro crioIlo'. Este representa el temor de la gente a que se propague de nuevo la guerra, con su secuela de calles ensangrentadas y niños sacrificados.

Es cierto que estamos en paz, pero el 'centauro criollo' nos advierte que el fantasma de la guerra aún anda cabalgando por nuestros campos como una amenaza terrible. El 'centauro criollo' es una proyección imaginaria, aunque nacida desde el fondo de nuestra construcción psíquica mestiza. En él cabalga corporizado nuestro atávico terror de volver hacia un pasado que signifique cascos aplastando cabezas y seres monstruosos devorando corazones de niños.

Ese duende —que viajó de un lado para otro del país pero que, por ahora, se ha detenido sintomáticamente en La Paz- es una creación colectiva. Es el alma doliente - judio errante- de todos los salvadoreños hartos ya de guerта, pero, sobre todo, hartos de injusticias y de desangramientos.

EJ 'centauro criollo' encama mágicamente para los salvadoreños el temor de que se frustren para siempre sus esperanzas de habitar en un pais hermoso, pero también digno y humano. Simbólicamente, pues, La Guerra aún sigue amenazando a La Paz" (p. 12).

\section{El demonio de la guerra en tierras de La Paz}

Es hora de hacer un análisis integrador del relato que está ante nosotros. Para este fin me ocuparé en refutar la hipótesis del "centauro criollo" de Rafael Rodríguez y retomaré las ideas surgidas de la aplicación de los métodos de Todorov y Lévi-Sırauss.

En primer lugar, una razón fuerte para sospechar de la validez del título de "centauro", asignado al hombre-caballo de nuestra historia, es el hecho de que en ningún momento es llamado de esa manera por los narradores autóctonos. Siempre se refieren a un "animal" que "De la cintura para'rriba es hombre, y de para'bajo..., es forma de caballo". Dejando de lado al compilador, sólo una informante usa la palabra "centauro" y se trata de la fuente más dudosa de todas: una alumna de la UCA que cuenta de oídas. Más difícil resulta sostener que un animal con las características señaladas - y en el contexto de los pobladores de 
la zona de las apariciones - obedezca a la proyección de un "arquetipo" cargado de todos esos elementos milológicos griegos, los cuales presuponen un mínimo conocimiento de dicha cultura... que no es precisamente el caso.

No hay que perder de vista el calificalivo de nuestro monstruo: el centauro es "criollo", porque en él no sólo hay un híbrido "bestia-humano" sino, además, "indígena-conquistador". Sobre este asunto, opino que se trata más bien de un recurso que Rafael Rodríguez emplea para obtener un elemento que sintetice tanto lo propio del "centauro" como el fenómeno de la violencia que tiene en la mira. Ahora bien, esto me parece forzar demasiado el "texto" en aras de un "significado" harto difícil de comprobar. Si nos atenemos al primero, la violencia -los sacrificios de niños-resulsa de un pacto con el Diablo y, al respecto, la tradición cristiana explica suficientemente lo de los sacrificios en un contexto diabólico y de brujería (v. gr. los "niños sin bautizar"). En otras palabras, no es necesario recurrir a la historia de los orígenes de los pueblos nonualcos y a la sobrevivencia de un "terror alávico" en sus descendientes; la escalera más corta nos lleva a las palabras mismas de los narradores: "dice ella que ha salido en la Biblia que se verán cosas muy malas (...) La gente dice que es cosa del diablo que anda ahl..., que el diablo hizo que apagaran las luces para que no le hicieran nada..." 9 .

Esto último nos permite enlazar con nuestros análisis desarrollados arriba. Tenemos primero el esquema quebranto de la ley $\rightarrow$ acción que busca evitar el castigo. Nos damos cuenta de inmediato que los sacrificios humanos son esa acción que busca revertir un castigo ya efectuado. Además, el pacto con el Diablo implica el desprecio de las leyes de Dios, que prohiben cualquier alianza con el Malo; pero también supone unas "leyes diabólicas", que en este caso fueron irrespetadas por el joven brujo al ser tan descuidado con su "alma" - irecordemos que la había dejado en un simple vaso con agua!-; esto le acarrea al joven su transformación en animal. Es así que el hombre-caballo de La Paz puede explicarse desde la presencia del Diablo - iel cual se presenta a los humanos con los cuartos traseros de equino!-; es el Maligno quien no sólo "castiga" al joven por sus descuidos, sino que, además, lo protege para que realice su tarea diabólica, persistiendo de esle modo en su pacto con él y en la transgresión de las leyes de Dios. Esto es lo que nos lleva a dejar abierto nuestro esquema de quebrantamiento de la ley, ya que la tarea que se realiza para evitar el castigo está instigada por el mismo Demonio, no por Dios".

"En la Biblia se verán cosas muy malas..." Los textos bíblicos que podrían relacionarse al relato del hombre-caballo echan bastante luz sobre la línea de interpretación - cuyo eje central es la violencia- que hemos elegido: los cuatro jinetes del Apocalipsis" son los portadores de la muerte y el exterminio de los hombres. Es el Demonio el que lleva la muerte a donde llega; para este fin utiliza a sus "jinetes" apocalípticos, los esbirros del Mal. No es necesaria dema- 
siada imaginación ni un forzamiento excesivo de la narración para ver en nuestro hombre-caballo a uno de estos hombres-a-caballo de los que habla la Escritura.

Y bien, ahora podemos reducir la relación pacto con el Diablo $\rightarrow$ sacrificios humanos a otra que nos permitirá agudizar aún más nuestra interpretación:

\section{ElMal $\rightarrow$ Muerte violenta.}

Si a esta relación añadimos la oposición que obteníamos del análisis del cuento a partir de las ideas de Lévi-Strauss:

\section{Lugareño — Muerte violenta,}

vernos que la muerte violenta no tiene otro agente que El Mal, el cual no pertenece a la esfera de lo cotidiano y lo "propio del lugar", sino que es "algo malo" que "viene de lejos". Nuestro esquema final sería:

\section{Lo extraño $\rightarrow$ Muerse violenta.}

Según la narración analizada, la violencia se interpreta como el resultado de ideas y costumbres que son ajenas al diario vivir de los lugareños. Esto parece coherente con la situación general del país en la época de las "apariciones", situación marcada por los recientes acuerdos de paz. A partir de estas fechas vemos que hay recurrencia en simplificar el problema de la violencia, responsabilizando a "otros países" de la guerra o reduciendo el problema de las "maras" a la influencia nociva de los deportados salvadoreños desde Estados Unidos. En todo caso, lo extraño, lo foráneo, se encuentra en el origen de los males que padece la sociedad ${ }^{12}$.

Lo anterior debería causamos preocupación, ya que refleja una lendencia que, lejos de desaparecer, se ha acentuado: me refiero al hecho de situar las causas de los conflictos sociales de El Salvador fuera de sus fronteras geográficas, económicas, culturales $e$ ideológicas. De las afirmaciones que sostienen, aún ahora, que la guenta obedeció exclusivamente a "la agresión comunisla dirigida a toda la América Latina", a los que dicen "nosotros queremos la paz, ellos quieren la violencia", no hay mucho trecho.

Estamos ante una interpretación maniquea del mundo y la sociedad, que encima afima que la maldad se encuentra en lo extraño y lo desconocido. " $E l$ otro es el enemigo". El extranjero, el que piensa de modo diferente, el que vive de modo diferente, ése es el malo, el demonio que quiere robarnos la paz. Por eso algunos aconsejan: "refugiémonos en lo mismo, en lo que nos pertenece desde lo más hondo". Lo cual, en otras palabras, quiere decir: "Seamos intolerantes con los diferentes". 
En el cuento aparece, en varias ocasiones, la idea de que la solución a las maldades que se cometen "allá afuera" se logra acudiendo a las fuentes del bien, que se identifican con la tradición campesina y cristiana de la zona. El agua bendita, la lectura de la Biblia, las buenas acciones y los rezos son elementos que hay que recuperar; son parte de la tradición que, según los narradores de la historia, se ha perdido. Por eso, me parece, en el cuento no hay una tendencia a ver el pasado con temor ${ }^{13}$, como podría suceder si se recuerdan las atrocidades de la guerra, sino que el pasado es ese universo idilico de paz que se les ha arrebatado. En la concepción del mundo de los lugareños se ve claramente el pasado como una categoría cósmica: pasado es el orden (cosmos) de paz que se ha perdido"4. Por su parte, el presente es el caos generalizado de una sociedad contemporánea corrompida y herida de muerte's.

Pero bien, la historia del hombre-caballo no sólo expresa angustias y temores sino, además, un esfuerzo por explicar las causas de los problemas. Expresa una profunda intuición popular, que no por plasmarse sobre unos esquemas tradicionalistas es peyorativa: la sifuación actual de crisis implica una ausencia de valores. La reflexión ética ulterior tal vez modifique la manera y los elementos esenciales del planteamiento, pero esto lo hará sobre la base de tal planteamiento, con lo cual se tiene ya un punto de partida comprensible para la misma comunidad.

Hay una idea que quiero recalcar para terminar estas reflexiones: esos maleficios que se suceden ahí en los montes de los Tepezontes y en los caminos de La Paz no son exclusivos de la localidad. Seguramente encontraríamos más de estos relatos en otros rincones de El Salvador, en el campo y en la misma ciudad capital. Es por eso que debemos estar a la escucha de lo que cuentan "nuestras gentes", para analizarlo y tratar de entenderlo. ¡No olvidemos que se trata de salvar nuestras vidas! De ahí la advertencia:

Bueno..., si les quiere decir a las demás gentes que tengan cuidado, porque no se sabe si va a volver... Tal vez a estos lugares porque están aislados... Pero nadie se tiene que confiar de esa cosa; porque supóngase que vaya a las ciudades. Allá es cierto que pasan las puertas cerradas..., pero también puede llegar...; no sólo aquí en el cantón...

Aquí han venido periodistas...; aquí han venido a preguntar gentes de otras partes, que si es cierto..., y nosotros les decimos que sí, porque sí es cierto.....

\section{Notas}

1. El estudio completo está inćdito.

2. Por identidad el autor entiende los elementos culturales y naturales que un individuo o comunidad posecn conscientemente, y que los definen, es decir, los describen, sólo en tanto estos elementos se encuentran en una relación de oposición respecto de los de otros individuos y comunidades, respectivamentc.

3. En el trabajo de Rafacl Rodrígucz, Los duendes de la barranca honda, el relato 
constituye el capítulo doce. "El centauro criollo" fue concluido el 8 de enero de 1994 y publicado en Co-Latino, "Suplemento cultural Tresmil", 22 de enero de 1994, pp. 10-12. En adelante deberá tomarse en cuenta que toda cita de este relato y los análisis del compilador corresponden a esa publicación. Las páginas se indican entre paréntesis.

4. Cfr. Tzvetan Todorov, La gramótica del Decamerón, Madrid, 1973. Este método sc detiene en la estructura de la trama y en el significado global que puede reducirse a un esquema universal. Las secuencias son las unidades semánticas pertinentes para entender el significado general del relato.

5. Cfr. Claude Lévi-Strauss, "La historia de Asdiwal", en Estructuralismo y antropologia, Buenos Aires, 1973. El autor se ocupa más bien de cómo el relato es la proyección literaria de un sentido global o conciencia, que el grupo ha conformado a partir de la interacción de elementos naturales (geografía, ecología, etc.) y culturales (normas sociales, cosmovision, etc.) propios de su contexto vital.

6. Se observan algunas contradicciones en el relato: no está claro si el monsiruo puede ser visto por alguien más que por los brujos; su transformación en persona para escapar de sus perseguidores, cuando antes se dice que la razón de los sacrificios es el retomo a su forma original; el informante que es identificado como "un señor" se transforma gradualmente en un jovencito dependiente de su "mami". No obstante, creo que estos problemas son en realidad insignificantes para la comprensión del sentido global del relato, ya porque en sí mismos no lo modifican, como por el hecho de que podríamos esperar que alguna investigación ulterior los resuelva o explique favorablemente.

7. La referencia a formas de vida y tradiciones cristianas se entiende en el sentido de cultura cristiana o cristiandad, de clara connotación católica. Ahora bien, en nuestro caso sería muy arriesgado hablar de "pensamiento católico" dada la presencia de otras iglesias cristianas y sectas en la zona, y a su posible influencia en la configuración de la "mentalidad" regional.

8. En todo caso se trataría de un "centauro mestizo", ya que se refiere al proceso de surgimiento de una conciencia ladina en nuestras lierras.

9. Por otra parte, es interesante el hecho de que el hombre-caballo recite pasajes de la Biblia referidos a los niños. La sentencia de la que habla Rafacl Rodríguez es probablemente la que se encuentra en la escena narrada en Le 18, 15-17, MI 19, 13-15 y Mc 10, 13-16, sólo que en tono sarcástico: "Dejad que los nin̄os vengan a mí..."

10. Es de soponer que la apertura es "radical", pues Dios no podría permitir que el Diablo triunfase, jaunque tampoco puede evitar que el malcficio continúc!

11. Cfr. Ap 6, 1-8.

12. Vale la pena recordar que, durante la década de los ochentas, la opinión pública salvadoreña tendía a ubicar geográlicamentc a la guerra civil en "cl oriente del país" (¿Usulután!) y en "las montañas del norte" (Chalatenango).

13. Esta es la interpretación de Rafael Rodríguez.

14. Este "pasado" no tiene nada que ver con el sistema cronológico cotidiano, ya que se trata, en cierto modo, de un tiempo moral-ontológico, una categoría que designa valores morales y metafisicos, y no fechas o "sucesos históricos". Se trata de un tiempo antes del tiempo.

15. Nótese la perfecta sintonía con la cosmovisión cristiana tradicional, la cual sostiene que El Mal procede de una calda, de una transgresión del orden Bueno. 